\title{
Changement climatique et forêts dans le Bassin du Congo Synergies entre l'adaptation et l'atténuation
}

\section{Contexte}

Face au changement climatique, deux grands types de stratégies sont disponibles : I'atténuation et l'adaptation. L'atténuation s'attaque aux causes du problème, en limitant l'accumulation des gaz à effet de serre dans l'atmosphère. L'adaptation s'attaque aux effets, en aidant la société et les écosystèmes à faire face au changement du climat.

Les forêts contribuent à l'atténuation car elles stockent du carbone. Réduire la déforestation est une stratégie d'atténuation. Les forêts contribuent aussi à l'adaptation des populations, par exemple en régulant les rivières ou en fournissant des produits importants pour des communautés dont l'agriculture ou l'élevage sont affectés par le climat.

L'adaptation gagne de l'importance dans l'arène internationale tandis que les politiques nationales et les projets mettent du temps à émerger dans les pays en développement. De plus, les forêts et les populations forestières sont peu prises en compte dans les débats et politiques nationales sur l'adaptation. Par ailleurs, le Bassin du Congo a une expérience limitée sur la réduction des emissions dues à la déforestation et à la dégradation des forêts (REDD+). Enfin, même s'il est admis que le secteur forestier offre des opportunités de synergie entre l'adaptation et l'atténuation, à ce jour, peu d'expériences grandeur nature et peu de connaissances viennent le confirmer.

\section{Description du projet}

Pour combler ce vide, le Centre de recherche forestière Internationale (CIFOR) a initié en 2010 un projet intitulé 'Changement Climatique et Forêts dans le Bassin du Congo : Synergies entre l'Adaptation et l'Atténuation' (COBAM).

Ce projet de 5 ans, financé par la Banque Africaine de Développement dans le cadre du support à la Communauté Economique des Etats d'Afrique Centrale (CEEAC) pour la mise en œuvre du Programme d'Appui à la Conservation des Ecosystèmes du Bassin du Congo (PACEBCo), sera implémenté dans 6 pays d'Afrique Centrale et dans 3 paysages du PACEBCo (voir Figure 1).

\section{Objectifs du projet}

L'objectif général du projet est de développer une réponse appropriée à la vulnérabilité liée aux changements climatiques dans les paysages du Bassin du Congo. II s'agit de fournir aux preneurs de décisions, praticiens et communautés locales les informations, analyses et outils nécessaires pour concevoir et mettre en œuvre des politiques et des projets d'adaptation au changement climatique et de réduction des émissions de carbone dans les forêts, avec des impacts équitables et des co-bénéfices sur la réduction de la pauvreté, l'amélioration des services écosystémiques autres que le carbone, et la protection des modes d'existence et des droits locaux.

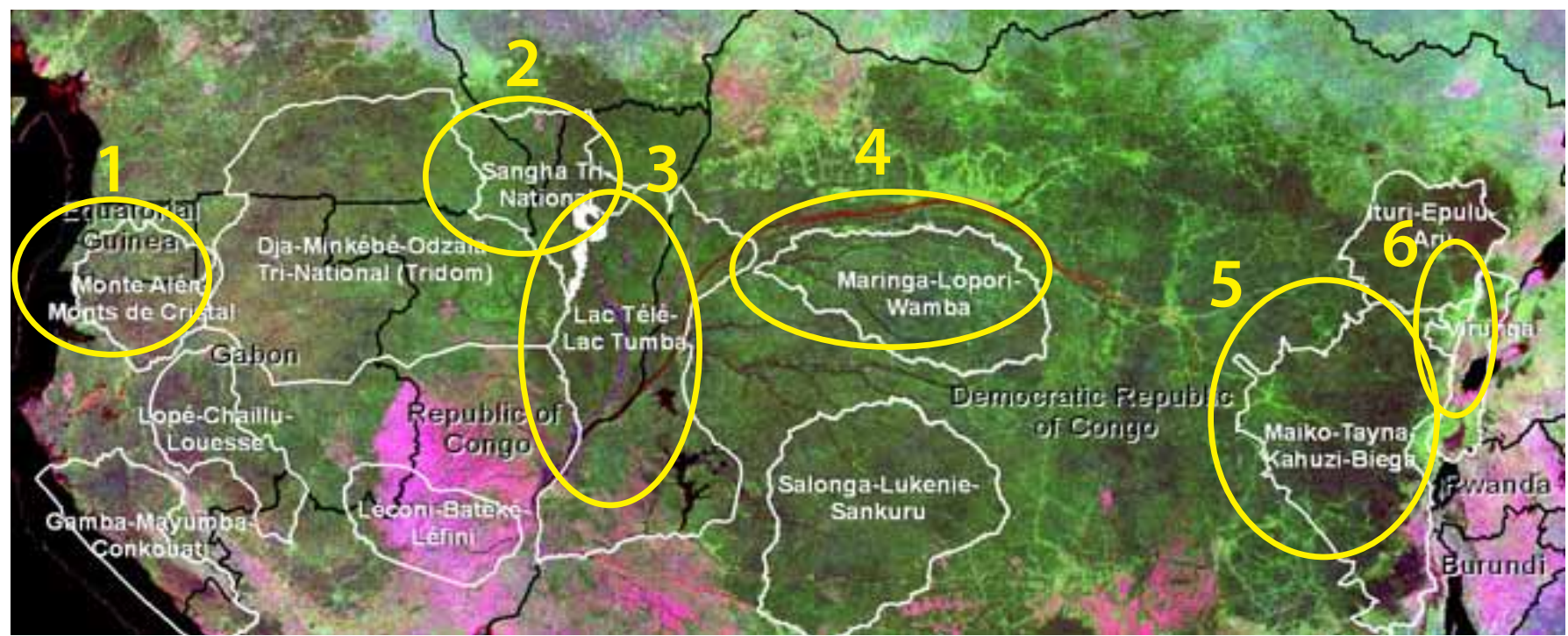

Figure 1. Les six paysages du Programme d'Appui pour la Conservation pour les Ecosystèmes du Bassin du Congo (PACEBCo) 


\section{Composantes de COBAM}

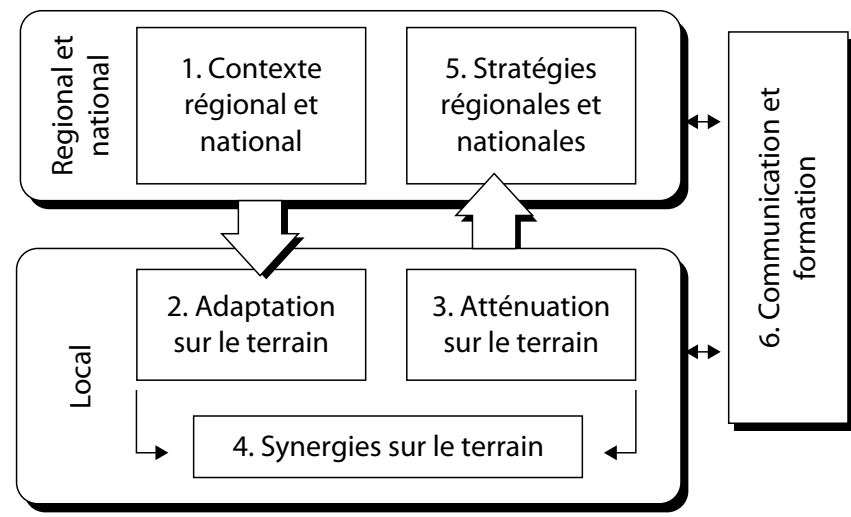

Figure 2. Organization de СОВАM

Les objectifs spécifiques sont:

1. Aider les acteurs dans la définition et la mise en œuvre de leurs propres mesures d'adaptation locale et de projets

REDD+, en se basant sur une analyse des politiques régionales et nationales relatives aux forêts et au changement climatique ;

2. Analyser la vulnérabilité des communautés locales et définir l'adaptation dans les sites sélectionnés;

3. Analyser les opportunités de la REDD+, les enjeux en termes de gouvernance et les impacts potentiels sur les modes d'existence locaux;

4. Recommander des approches pour définir des projets locaux renforçant les synergies entre atténuation et adaptation et identifier et mobiliser des partenariats pour la mise en œuvre de tels projets ;

5. Développer des stratégies nationales et régionales pour que l'adaptation et la REDD+ soient intégrés dans les politiques forestières et pour favoriser les synergies entre adaptation et atténuation ;

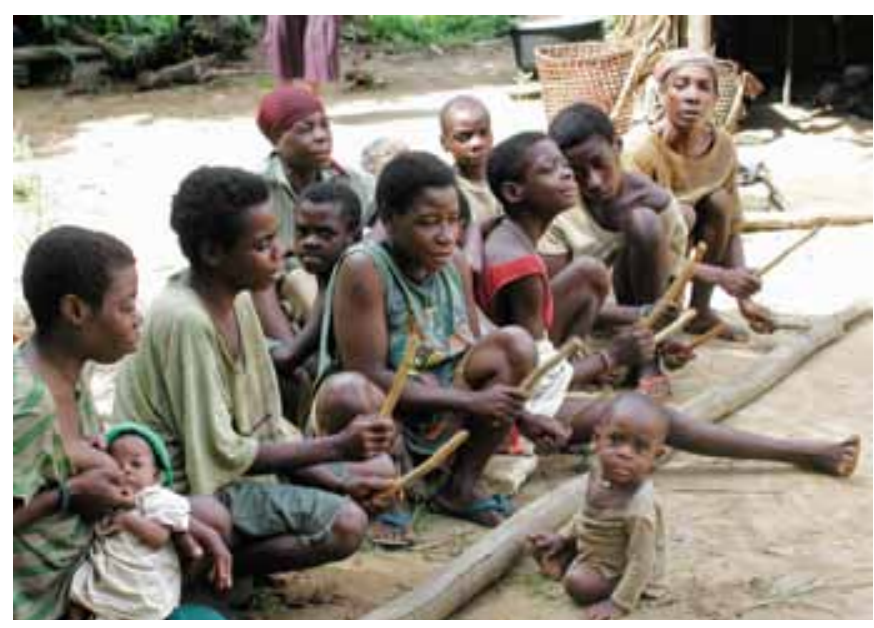

Les pygmées BaAka de la République centrafricaine, premières victimes de la déforestation

6. Communiquer, partager les connaissances et renforcer les capacités aux échelles locales, sous-nationales et nationales à propos de l'adaptation et REDD+ dans les forêts du Bassin du Congo.

\section{Partenariat autour de COBAM}

En plus du CIFOR, le projet COBAM fera intervenir plusieurs partenaires:

- Les centres de recherche et universités nationales en Afrique Centrale

- Des partenaires internationaux: CIRAD, SEI, UEA, IRD

- Des organisations régionales: COMIFAC, BAD, CEEAC, CARPE, PFBC, etc.

- Des ONGs présentes dans les sites

- D'autres projets du CIFOR : CoFCCA, GCS, FORAFAMA

\section{Contacts}

Anne Marie Tiani: a.tiani@cgiar.org

Youssoufa Bele : b.youssoufa@cgiar.org

Edith Abilogo: e.abilogo@cgiar.org

Bureau régional pour l'Afrique Centrale : cifor.cameroon@cgiar.org

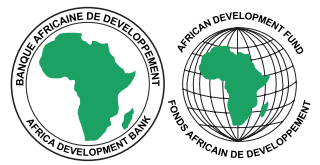

www.cifor.cgiar.org
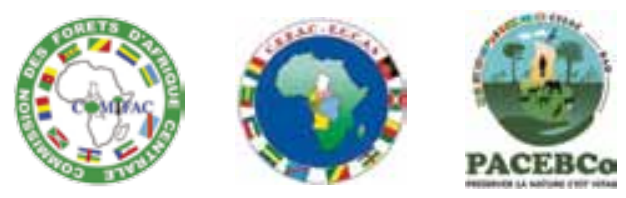

PACEBCo

\section{(WWW.cifor.cgiar.org} éclairer les politiques et les pratiques qui affectent les forêts dans les pays en développement. CIFOR est l'un des 15 centres au sein du Groupe consultatif sur la recherche agricole internationale (CGIAR). Le siège du CIFOR est situé à Bogor, en Indonésie. CIFOR a également des bureaux en Asie, en Afrique et en Amérique du Sud. 